\title{
Rituximab With High-Dose Methotrexate In Newly Diagnosed Primary Central Nervous System Lymphoma: a Single-Center Experience From China
}

\author{
Xianggui Yuan \\ Second Affiliated Hospital of Zhejiang University \\ Teng Yu \\ Second Affiliated Hospital of Zhejiang University \\ Yurong Huang \\ Second Affiliated Hospital of Zhejiang University \\ Huawei Jiang \\ Second Affiliated Hospital of Zhejiang University \\ Xiaohua Xu \\ Second Affiliated Hospital of Zhejiang University \\ Yun Liang \\ Second Affiliated Hospital of Zhejiang University \\ Wen-Bin Qian ( $\nabla$ qianwb@zju.edu.cn ) \\ Second Affiliated Hospital of Zhejiang University
}

\section{Research Article}

Keywords: Rituximab, Methotrexate, Primary central nervous system lymphoma, Cytarabine

Posted Date: January 7th, 2022

DOI: https://doi.org/10.21203/rs.3.rs-1204353/v1

License: (c) (i) This work is licensed under a Creative Commons Attribution 4.0 International License. Read Full License 


\section{Abstract}

Induction chemotherapy based on high-dose methotrexate is considered as the standard approach for newly diagnosed primary central nervous system lymphomas (PCNSLs). However, the best combination chemotherapeutic regimen remains unclear. This study aimed to determine the efficacy and toxicities of rituximab with methotrexate (R-M regimen). Consecutive 37 Chinese patients receiving R-M regimen as induction chemotherapy were retrospectively identified from January 2015 to June 2020 from our center in eastern China. Fourteen patients receiving rituximab plus methotrexate with cytarabine (R-MA regimen) at the same period were identified as the positive control group. The response rates, survival, toxicities, length of hospital stay (LOS), and cost were compared. Compared with the R-MA regimen, the R-M regimen showed comparable response rate and survival outcomes, but had fewer grade 3-4 hematological toxicities, shorter LOS, lower mean total hospitalization cost and lower mean total antibiotic cost. Overall response after two cycles of chemotherapy, complete remission at the end of induction chemotherapy and ECOG>3 were independent prognostic factors for overall survival. In conclusion, R-M regimen is an effective and well-tolerated combination treatment for PCNSLs, which warrants further evaluation in randomized trials.

\section{Introduction}

Primary central nervous system lymphoma (PCNSLs) is a rare and aggressive extranodal non-Hodgkin lymphoma located in the brain, leptomeninges, spinal cord, eyes or cerebrospinal fluid (CSF) ${ }^{1}$. PCNSLs are characterized by short-lasting responses and poor prognosis. High-dose methotrexate (HD-MTX) is the standard treatment for newly diagnosed PCNSLs. However, the efficacy of HD-MTX monotherapy is unsatisfactory with a median overall survival (OS) of 16-38 months and progression-free survival (PFS) of 4-10 months ${ }^{2-4}$. As such, other drugs in combination with HD-MTX have been investigated to improve the outcome ${ }^{5}$. Cytarabine (Ara-C) is a cell cycle-phase specific antimetabolite, commonly used in chemotherapy for hematologic malignancies. IELSG20, a phase 2 trial, indicated that the combination of Ara-C with HD-MTX increased the CR rate (46\% vs. $18 \%$ ) and improved the 3 -year OS (46\% vs. 32\%) in PCNSLs ${ }^{4}$. IELSG32, another phase 2 trial, revealed that patients receiving the HD-MTX plus Ara-C (MA regimen) together with thiotepa and rituximab (MATRix) had a higher CR rate (49\% vs. 23\%) and improved PFS and OS, than those receiving the MA regimen alone ${ }^{6}$. Although combination chemotherapeutic regimens bring increasing efficacy, hematological toxicities are the primary concerns. Therefore, the best combination chemotherapeutic regimen remains unclear.

Rituximab, a highly specific monoclonal antibody against CD20, is widely used to treat diffuse large $B$ cell lymphoma (DLBCL). The combination of rituximab with the cyclophosphamide, doxorubicin, vincristine and prednisone (R-CHOP) regimen has been proved to significantly improve the OS of systemic DLBCL ${ }^{7}$. Although rituximab's clinical effect in PCNSLs remains controversial due to its large size and poor penetration of the blood-brain barrier (BBB), several preliminary studies suggest that adding rituximab to cytotoxic chemotherapy is safe and effective for PCNSLs ${ }^{2,8,9}$. Studies have indicated that rituximab is likely to reach therapeutic concentrations and can induce responses in contrast-enhanced lesions, in which there is a substantial disruption of the $\mathrm{BBB}^{10,11}$. Two randomized controlled trials were designed to formally determine the effects of rituximab on PCNSLs. The IELSG32 study stated that the addition of rituximab to the MA regimen (R-MA regimen) was more likely to induce a response than the MA regimen alone (ORR,74\% vs. 53\%) and there was a trend for improved PFS ( $p$ value 0.051 ) and OS ( $p$-value 0.095) with overall minimal added toxicities ${ }^{6}$. However, the HOVON 105/ALLG NHL 24 study, a phase 3 randomized study, did not find significantly increased PFS, or OS from the addition of rituximab to the MTX, carmustine, teniposide, and prednisolone (MBVP) chemotherapy regimen compared with the MBVP regimen alone in newlydiagnosed PCNSLs ${ }^{12}$, which was contrary to the results in the IELSG32 study. Interestingly, subgroup analysis in the HOVON105 study showed that the application of rituximab might benefit patients under 60 years of age. Even with the conflicting results of the above two prospective randomized studies, given the relatively low toxicities and single-agent activity of rituximab in relapsed/refractory PCNSLs, rituximab has been widely used in clinical practice and incorporated into clinical trials ${ }^{13}$. 
To balance therapy intensification with side effects in our center, rituximab with methotrexate (R-M regimen) is widely applied for PCNSLs. This study aimed to retrospectively evaluate the efficacy and toxicities of the R-M regimen as induction chemotherapy for newly diagnosed PCNSLs.

\section{Patients And Methods}

\section{Patients}

From January 2015 to June 2020, consecutive patients with newly diagnosed PCNSLs, receiving the R-M regimen as induction therapy at the Second Affiliated Hospital, Zhejiang University, were identified from medical records. Given the inaccessibility of thiotepa in China, the R-MA regimen but not the MATRix regimen was widely applied since the IELSG32 results was first published in 2016. Thus, patients receiving the R-MA regimen at the same period were identified as the positive control group. Patients fulfilling the following criteria were enrolled in the study: newly diagnosed patients with PCNSL, definite pathological diagnosis of DLBCL, and measurably enhanced lesions for response assessment. Patients with isolated ocular lymphomas or HIV- associated lymphoma were excluded. Informed consent was obtained from all patients enrolled in this study. According to the updated WHO classifications, the diagnosis of PCNSLs was confirmed by two pathologists and consensus was reached for each patient. This study was approved by the Ethics Committee of the Second Affiliated Hospital, Zhejiang University.

\section{Treatment protocol}

Patients were scheduled to receive 6 cycles of induction chemotherapy on a 21-day cycle. Thereafter, consolidation chemotherapy with whole-brain radiation therapy (WBRT,30-36 Gy followed by a limited field to gross disease to 45 Gy) was administered, depending on their age, financial situation and willingness. Patients who failed to reach PR after 4 courses of induction chemotherapy or progressed at any time were withdrawn and received salvage treatment. The R-M regimen consisted of rituximab and MTX, while the R-MA regimen consisted of rituximab, MTX and Ara-C. Rituximab (375 mg/m2) was administered on day 0. HD-MTX $(3.5 \mathrm{~g} / \mathrm{m} 2)$ was administered intravenously over 4 hours on day 1 . Patients received adequate pre-and post-MTX hydration, urinary alkalization and leucovorin rescue. Leucovorin rescue was started 14 hours after completing HD-MTX infusion and administered at $15 \mathrm{mg}$ every 6 hours until serum MTX levels were $\leq 0.05 \mu \mathrm{mol} / \mathrm{l}$. Ara-C $(2 \mathrm{~g} / \mathrm{m} 2)$ was administered intravenously every 12 hours on days 2-3. All patients who had grade 3-4 neutropenia with infection received prophylactic lenograstim after each cycle and the dose of Ara-C was reduced thereafter.

\section{Response evaluation}

Treatment responses were evaluated every 2 induction chemotherapy cycles or at any time the neurological symptoms worsened or re-emerged by brain magnetic resonance imaging (MRI) with contrast enhancement. The responses were classified as complete remission (CR), unconfirmed complete remission (CRu), partial remission (PR), stable disease (SD), or progression disease (PD), according to the International Primary CNS Lymphoma Collaborative Group (IPCG) criteria ${ }^{14}$. For statistical analysis, CR and CRu defined by the IPCG criteria were combined into a single "CR" category previously defined by Response Evaluation Criteria in Solid Tumors in this study. Overall response=CR+PR. After completing the treatment, patients were assessed by repeated enhanced brain MRI every 3 months for the first 2 years and every 6 months for the next 3 years. OS was determined as the period from the date of diagnosis of PCNSLs to death from any cause or the last follow-up. PFS was defined as the time from diagnosis to disease progression or death due to PCNSL or last follow-up.

\section{Determination of length of hospital stay (LOS)}

LOS was calculated from the day of chemotherapy to discharge and recorded in whole days. When a case was readmitted for side effects, the duration was added to the initial LOS. LOS was calculated for the R-M group and R-MA group, respectively. 


\section{Cost analysis}

The cost of hospital stays and antibiotics was determined from patient billing records and converted to US dollars at the currency exchange rate at the time of this study. The cost of hospital stays and antibiotics were calculated for the R-M group and R-MA group, respectively.

\section{Toxicity evaluation}

Treatment toxicities were evaluated according to the National Cancer Institute Common Toxicity Criteria (CTCAE) version 4.0(https:// evs.nci.nih.gov/ftp1/CTCAE /CTCAE_ 4.03).

\section{Statistical analysis}

Continuous variables were summarized as means with $95 \%$ confidence intervals $(95 \% \mathrm{Cls})$ and compared with an unpaired ttest. Categorical variables were summarized as frequencies with associated percentages. The patient characteristics and treatment responses of the two therapeutic groups were compared using the chi-square test or Fisher's exact test. The OS and PFS were calculated with the Kaplan-Meier method and compared with the log-rank test. The multivariate analysis for OS and PFS was performed based on the Cox proportional hazards regression model. The results were expressed as hazard ratios (HR) and their $95 \% \mathrm{Cls}$. Those factors with p-values less than 0.1 in univariate analysis were used to construct multivariate models for OS and PFS. For continuous variables, the best-identified cutoff point was determined by receiver operating characteristic (ROC) curve analysis. All statistical analyses were performed using SPSS v17.0 for Windows (SPSS Inc, Chicago, IL). An alpha value of two-sided $p<0.05$ was considered statistically significant.

All above methods were performed in accordance with the relevant guidelines and regulations.

\section{Results}

\section{Patient characteristics and treatment}

A total of 34 patients receiving R-M regimen were enrolled. The diagnosis was achieved by surgery (40.5\%), or stereotactic biopsy (59.5\%), and no patients were diagnosed based on CSF. All PCNSLs were confirmed to be DLBCL. The median age of patients was 62 years (range, 27 to 80 years; 23 patients were $>60$ years old, and 14 were $\leq 60$ years old). Five patients (13.5\%) had elevated LDH above the upper limit of normal. 77.1\% (27/35) patients had non-germinal center B cell-like (nonGCB) DLBCL. Severn (20.6\%) patients were consolidated with WBRT.

Fourteen patients receiving the R-MA regimen as induction chemotherapy were identified as the positive control group. The characteristics of the 51 patients are described in Table 1. These patients were treated according to their age, financial situation and willingness. The demographics and baseline clinical characteristics (sex, ECOG, LDH, deep structure involvement, biopsy type, Karnofsky performance status (KPS), CD10, C-Myc, Bcl-2, Bcl-6, MUM-1, pathological phenotype, and CSF protein) were not different between patients treated with the R-M and R-MA regimens, except for the age and number of lesions. Patients in the R-M group were older $(p=0.006)$ than the R-MA group, and more patients in the R-M group had multiple lesions $(p=0.024)$. 
Table 1

Baseline patient characteristics and the distribution of lymphoma subtypes

\begin{tabular}{|c|c|c|c|c|c|}
\hline Factors & & Total(n=51) & $R-M(n=37)$ & $R-M A(n=14)$ & P-value \\
\hline \multirow[t]{3}{*}{ Age (year) (median, range) } & & $60(27-80)$ & $62(27-80)$ & $56(41-64)$ & 0.012 \\
\hline & $>60 y$ & $25(49.0 \%)$ & $23(62.2 \%)$ & $2(14.3 \%)$ & \\
\hline & $\leq 60 y$ & $26(51.0 \%)$ & $14(37.8 \%)$ & $12(85.7 \%)$ & 0.002 \\
\hline \multirow[t]{2}{*}{ Gender } & Male & $28(54.9 \%)$ & $22(59.5 \%)$ & $6(42.9 \%)$ & \\
\hline & Female & $23(45.1 \%)$ & $15(40.5 \%)$ & $8(57.1)$ & 0.29 \\
\hline \multirow[t]{2}{*}{ Multifocal lesions } & Yes & $24(47.1 \%)$ & $21(56.8 \%)$ & $3(21.4 \%)$ & \\
\hline & No & $27(52.9 \%)$ & $16(43.2 \%)$ & $11(78.6 \%)$ & 0.024 \\
\hline \multirow[t]{2}{*}{ Deep-brain involvement } & Present & $31(60.8 \%)$ & $22(59.5 \%)$ & $9(64.3 \%)$ & \\
\hline & Absent & $20(39.2 \%)$ & $15(40.5 \%)$ & $5(35.7 \%)$ & 0.75 \\
\hline \multirow[t]{2}{*}{ Biopsy type } & Surgical & $21(41.2 \%)$ & $15(40.5 \%)$ & $6(42.9 \%)$ & \\
\hline & Stereotactic & $30(58.8 \%)$ & $22(59.5 \%)$ & $8(57.1 \%)$ & 0.88 \\
\hline \multirow[t]{4}{*}{ ECOG score } & 1 & $0(0 \%)$ & $0(0 \%)$ & $0(0 \%)$ & \\
\hline & 2 & $26(51.0 \%)$ & $17(45.9 \%)$ & $9(64.3 \%)$ & \\
\hline & 3 & $15(29.4 \%)$ & $12(32.4 \%)$ & $3(21.4 \%)$ & \\
\hline & 4 & 10(19.6\%) & $8(21.6 \%)$ & $2(14.3 \%)$ & 0.51 \\
\hline \multirow[t]{2}{*}{ KPS score } & $<70$ & $32(62.7 \%)$ & $24(64.9 \%)$ & $8(57.1 \%)$ & \\
\hline & $\geq 70$ & 19(37.3\%) & $13(35.1 \%)$ & $6(42.9 \%)$ & 0.61 \\
\hline \multirow[t]{2}{*}{ LDH } & Elevated & $7(13.7 \%)$ & $5(13.5 \%)$ & $2(14.3 \%)$ & \\
\hline & Normal & $44(86.3 \%)$ & $32(86.5 \%)$ & 12(85.7\%) & 1.00 \\
\hline \multirow[t]{2}{*}{ CSF protein } & Elevated & $22 / 34(64.7 \%)$ & $16 / 25(64.0 \%)$ & $6 / 9(66.7 \%)$ & \\
\hline & Normal & 12/34(35.3\%) & $9 / 25(36.0 \%)$ & $3 / 9(33.3 \%)$ & 1.00 \\
\hline \multirow[t]{2}{*}{ C-Myc } & Negative & $24 / 45(53.3 \%)$ & 17/32(53.1\%) & 7/13(53.8\%) & \\
\hline & Positive & $21 / 45(46.7 \%)$ & $15 / 32(46.9 \%)$ & 6/13(46.2\%) & 0.96 \\
\hline \multirow[t]{2}{*}{$\mathrm{Bcl}-2$} & Negative & $16 / 47(34.0 \%)$ & 12/34(35.3\%) & 4/13(30.8\%) & \\
\hline & Positive & $31 / 47(66.0 \%)$ & $22 / 34(64.7 \%)$ & $9 / 13(69.2 \%)$ & 1.00 \\
\hline \multirow[t]{2}{*}{$\mathrm{Bcl}-6$} & Negative & $10 / 49(20.4 \%)$ & $8 / 36(22.2 \%)$ & $2 / 13(15.4 \%)$ & \\
\hline & Positive & $39 / 49(79.4 \%)$ & $28 / 36(77.8 \%)$ & $11 / 13(84.6 \%)$ & 0.71 \\
\hline \multirow[t]{2}{*}{ CD10 } & Negative & $38 / 49(77.6 \%)$ & $29 / 36(80.6 \%)$ & $9 / 13(69.2 \%)$ & \\
\hline & Positive & $11 / 49(22.4 \%)$ & 7/36(19.4\%) & 4/13(30.8\%) & 0.40 \\
\hline MUM-1 & Negative & $7 / 48(14.6 \%)$ & $7 / 35(20 \%)$ & $0 / 13(0 \%)$ & \\
\hline
\end{tabular}

Notes: CSF, cerebrospinal fluid; ECOG, Eastern Cooperative Oncology Group; GCB, germinal center B-cell-like; KPS, Karnofsky performance status; $L D H$, lactate dehydrogenase; $R-M$, combination regimen of high-dose methotrexate and rituximab; R-MA, combination regimen of rituximab, high-dose methotrexate and cytarabine. 


\begin{tabular}{|llllll|}
\hline Factors & & Total(n=51) & R-M(n=37) & R-MA(n=14) & P-value \\
\hline & Positive & $41 / 48(85.4 \%)$ & $28 / 35(80 \%)$ & $13 / 13(100 \%)$ & 0.17 \\
\hline Pathology phenotype & Non-GCB & $35 / 48(72.9 \%)$ & $27 / 35(77.1 \%)$ & $8 / 13(61.5 \%)$ & 0.28 \\
\cline { 2 - 5 } & GCB & $13 / 48(27.1 \%)$ & $8 / 35(22.9 \%)$ & $5 / 13(38.5 \%)$ & \\
\hline $\begin{array}{l}\text { Notes: CSF, cerebrospinal fluid; ECOG, Eastern Cooperative Oncology Group; GCB, germinal center B-cell-like; KPS, } \\
\text { Karnofsky performance status; LDH, lactate dehydrogenase; R-M, combination regimen of high-dose methotrexate and } \\
\text { rituximab; R-MA, combination regimen of rituximab, high-dose methotrexate and cytarabine. }\end{array}$ & \\
\hline
\end{tabular}

\section{Response and survival}

Thirty-seven patients received a total of 184 cycles of the R-M regimen with a median of 6 cycles per patient (range, 1-6). At the last follow-up, 20 achieved CR (54.1\%), 8 achieved PR (21.6\%), 0 had SD (0\%), and 9 had PD (24.3\%). Fourteen patients received a total of 39 cycles of the R-MA regimen, with a median of 3 cycles per patient (range, 1-6). At the last follow-up, 5 patients achieved CR (35.7\%), 2 achieved PR (14.3\%), 1 had SD (7.1\%), and 6 had PD (42.9\%). The R-M regimen produced a comparable CR rate $(54.1 \%$ vs. $35.7 \%, p=0.24)$ and overall response rate (ORR) $(75.6 \%$ vs. $50.0 \%, p=0.078)$, compared with the R-MA regimen (Figure 1A).

Follow-up data were available for all patients in both groups. The median follow-up duration was 30.0 months (range,1-76) in the R-M group, which was similar to 27.5 months (range, 1-51) in the R-MA group ( $p=0.44)$. The median OS was not reached in either cohort with estimated 3-year OS rates of $54.4 \%$ and $51.9 \%$ in the R-M and R-MA group, respectively. The median PFS of the R-M group was 19.0 months $(95 \% \mathrm{Cl}, 16.0-22.0)$ and the median PFS of R-MA group was 9.0 months $(95 \% \mathrm{Cl}, 0-27.3)$. The estimated 2-year PFS rates were36.6\% and $42.9 \%$ in the R-M and R-MA groups, respectively. The PFS (Figure 1B).and OS (Figure 1C) for the R-M group were not inferior to the R-MA group ( $p=0.94, p=0.85$, respectively.).

\section{Toxicity and cost}

Thirty-seven patients received a total of 184 cycles of the R-M regimen with a median of 6 cycles per patient (range, 1-6). Twenty-four of the 37 patients completed the entire protocol. Only 1 patient (2.7\%) was withdrawn due to severe myelosuppression with infection and 9 patients (24.3\%) were withdrawn due to disease progression. The remaining 3 patients were withdrawn due to personal reasons. Fourteen patients received a total of 39 cycles of the R-MA regimen, with a median of 3 cycles per patient (range, 1-6). Only three of the 14 patients completed the entire protocol. Indeed, five patients (35.7\%) were withdrawn due to severe myelosuppression with infection, and six patients $(42.9 \%)$ were withdrawn due to the disease progression. The total chemotherapy completion rate was higher in the R-M group than in the R-MA group (64.9\% vs. $21.4 \%, p=0.006$ ) (Figure 1D). The chemotherapy interruption rate due to toxicities was significantly lower in the R-M group than in the R-MA group (2.7\% vs. $35.7 \%, p=0.004)$.

The most frequent toxicities were hematologic toxicities and infection, as summarized in Table 2. The R-M group experienced significantly fewer frequent grade $3-4$ hematological toxicities $(3.4 \%$ vs. $59.0 \%, p<0.001)$ and febrile neutropenia $(2.7 \%$ vs. $41.0 \%, p<0.001)$ than the R-MA group. Patients in the R-M group experienced significantly less frequent grade 3 neutropenia ( $1.6 \%$ vs. $15.4 \%, p=0.001)$, grade 4 neutropenia ( $1.1 \%$ vs. $43.6 \%, p<0.001)$, grade 3 anemia $(0.5 \%$ vs. $12.8 \%, p=0.001)$, grade 4 anemia ( $0 \%$ vs. $10.3 \%, p=0.001)$ and grade 4 thrombopenia $(1.1 \%$ vs. $51.3 \%, p<0.001)$ than the R-MA group. Urinary tract infections $(1.6 \%$ vs. $17.9 \%, p<0.001)$ and pneumonia $(1.6 \%$ vs. $17.9 \%, p<0.001)$ were also observed much less frequently in the R-M group than in the R-MA group. Other nonhematological toxicities were generally mild to moderate and reversible and occurred at similar frequencies across both treatment groups. No treatment-related deaths were observed in either group. There were 2 cases of atrial fibrillation, one case of deep vein thrombosis, one case of acute heart failure, and one case of grade 4 skin rash in the R-MA group. 
Table 2

Main adverse effects by treatment group

\begin{tabular}{|c|c|c|c|}
\hline Toxicities & R-M ( $n=184$ cycles) & R-MA ( $n=39$ cycles) cecs) & P-value \\
\hline Grade 3 neutropenia & $3(1.6 \%)$ & $6(15.4 \%)$ & 0.001 \\
\hline Grade 4 neutropenia & $2(1.1 \%)$ & $17(43.6 \%)$ & $<0.001$ \\
\hline Grade 3 thrombopenia & $4(2.2 \%)$ & $3(7.7 \%)$ & 0.10 \\
\hline Grade 4 thrombopenia & $2(1.1 \%)$ & $20(51.3 \%)$ & $<0.001$ \\
\hline Grade 3 anemia & $1(0.5 \%)$ & $5(12.8 \%)$ & 0.001 \\
\hline Grade 4 anemia & $0(0 \%)$ & $4(10.3 \%)$ & 0.001 \\
\hline Febrile neutropenia & $5(2.7 \%)$ & $16(41.0 \%)$ & $<0.001$ \\
\hline Urinary tract infection & $3(1.6 \%)$ & $7(17.9 \%)$ & $<0.001$ \\
\hline Pneumonia & $3(1.6 \%)$ & $7(17.9 \%)$ & $<0.001$ \\
\hline Oral candidiasis & $3(1.6 \%)$ & $0(0 \%)$ & 1.00 \\
\hline Sepsis & $1(0.5 \%)$ & $1(2.6 \%)$ & 0.32 \\
\hline Acute infectious enteritis & $1(0.5 \%)$ & $0(0 \%)$ & 1.00 \\
\hline Herpes zoster & $1(0.5 \%)$ & $0(0 \%)$ & 1.00 \\
\hline Grade 3 Hepatotoxicity & $3(1.6 \%)$ & $3(7.7 \%)$ & 0.068 \\
\hline Grade 4 Hepatotoxicity & $1(0.5 \%)$ & $0(0 \%)$ & 1.00 \\
\hline Creatinine elevated & $2(1.1 \%)$ & $0(0 \%)$ & 1.00 \\
\hline Deep vein thrombosis & $0(0 \%)$ & $1(2.6 \%)$ & 0.18 \\
\hline Atrial fibrillation & $0(0 \%)$ & $2(5.1 \%)$ & 0.03 \\
\hline Acute heart failure & $0(0 \%)$ & $1(2.6 \%)$ & 0.18 \\
\hline Grade 4 skin rash & $0(0 \%)$ & $1(2.6 \%)$ & 0.18 \\
\hline
\end{tabular}

The average LOS was 5.7 days (95\% Cl: 5.3-6.1) in the R-M group, which was shorter than 15.2 days (95\% $\mathrm{Cl}$ : 12.7-17.3) observed in the R-MA group ( $\mathrm{p}<0.001$ ) (Figure 1E). The mean total hospitalization cost per cycle of the R-M regimen per patient was $\$ 5,536$ (95\% Cl: 4,830-6,454), with a mean total antibiotic cost per cycle per patient of \$157 (95\%Cl: $37-291)$. The mean total hospitalization cost per cycle of the R-MA regimen per patient was $\$ 8,741$ (95\%Cl: 6,904-10,352), with a mean total antibiotic cost per cycle per patient of $\$ 822(95 \% \mathrm{Cl}: 417-1,185)$. The mean total hospitalization cost (Figure 1F). and mean total antibiotic cost per cycle per patient (Figure 1G) in the R-MA group were 1.58 times $(P=0.0009)$ and 5.24 times $(P=0.0026)$ that of the R-M group, respectively.

\section{Univariate and multivariate analyses of prognostic indicators}

The results of univariate and multivariate analyses of prognostic indicators are summarized in Table 3. The univariate analysis indicated that response of overall response after two cycles of chemotherapy $(p=0.000)$, complete remission at the end of the induction therapy $(p=0.002)$ and overall response at the end of the induction therapy $(p=0.000)$ and GCB subtype $(p=0.019)$ were associated with increased PFS (Figure 2A-D). While overall response after two cycles of chemotherapy $(p=0.003)$, complete remission at the end of the induction therapy $(p=0.01)$, overall response at the end of the induction therapy $(p=0.004)$, age $>58(p=0.041)$ and ECOG $>3(p=0.007)$ were associated with increased OS (Figure 2E-H). Disappointingly, we did not observe any association of LDH or deep brain involvement with PFS or OS. We incorporated all 
those factors with $\mathrm{p}$-values $<0.1$ in univariate analysis into the multivariate Cox analysis and revealed that overall response after two cycles of induction chemotherapy $(p=0.073)$,complete remission at the end of induction chemotherapy $(p=0.070)$ and ECOG >3 ( $p=0.007)$ were independent prognostic factors for OS. While overall response at the end of induction chemotherapy $(p=0.049)$ and multifocal $(p=0.067)$ were identified as dependent prognostic indicators for PFS.

Table 3

Univariate and multivariate analyses of overall and progression-free survival

\begin{tabular}{|c|c|c|c|c|c|c|c|c|c|c|c|c|}
\hline \multirow{3}{*}{ Variable } & \multicolumn{6}{|l|}{ PFS } & \multicolumn{6}{|l|}{ os } \\
\hline & \multicolumn{3}{|c|}{ Univariate analysis } & \multicolumn{3}{|c|}{ Multivariate analysis } & \multicolumn{3}{|c|}{ Univariate analysis } & \multicolumn{3}{|c|}{ Multivariate analysis } \\
\hline & $\mathrm{HR}$ & $95 \% \mathrm{Cl}$ & $\begin{array}{l}\mathrm{P}- \\
\text { value }\end{array}$ & $\mathrm{HR}$ & $95 \% \mathrm{Cl}$ & $\begin{array}{l}\mathrm{P}- \\
\text { value }\end{array}$ & $\mathrm{HR}$ & $95 \% \mathrm{Cl}$ & $\begin{array}{l}\mathrm{P}- \\
\text { value }\end{array}$ & $\mathrm{HR}$ & $95 \% \mathrm{Cl}$ & $\begin{array}{l}\mathrm{P} \text { - } \\
\text { value }\end{array}$ \\
\hline Age $>58 y$ & 1.47 & $0.71-3.05$ & 0.299 & & & & 2.83 & $\begin{array}{l}1.04- \\
7.69\end{array}$ & 0.041 & 2.42 & $\begin{array}{l}0.85- \\
6.86\end{array}$ & 0.097 \\
\hline $\begin{array}{l}\text { Deep-brain } \\
\text { involvement }\end{array}$ & 1.11 & $0.55-2.25$ & 0.755 & & & & 0.90 & $\begin{array}{l}0.38- \\
2.11\end{array}$ & 0.805 & & & \\
\hline$E C O G>3$ & 1.94 & $0.89-4.20$ & 0.095 & 1.80 & $\begin{array}{l}0.73- \\
4.42\end{array}$ & 0.202 & 2.83 & $\begin{array}{l}1.15- \\
7.00\end{array}$ & 0.024 & 4.85 & $\begin{array}{l}1.54- \\
15.25\end{array}$ & 0.007 \\
\hline $\begin{array}{l}\text { Elevated } \\
\text { LDH }\end{array}$ & 1.12 & $0.53-2.38$ & 0.764 & & & & 1.73 & $\begin{array}{l}0.58- \\
5.12\end{array}$ & 0.323 & & & \\
\hline $\begin{array}{l}\text { Multiple } \\
\text { focal }\end{array}$ & 1.84 & $0.92-3.66$ & 0.083 & 2.12 & $\begin{array}{l}0.95- \\
4.76\end{array}$ & 0.067 & 1.49 & $\begin{array}{l}0.64- \\
3.45\end{array}$ & 0.353 & & & \\
\hline $\begin{array}{l}\text { GCB } \\
\text { subtype }\end{array}$ & 0.24 & $0.07-0.79$ & 0.019 & 0.35 & $\begin{array}{l}0.10- \\
1.22\end{array}$ & 0.100 & 0.28 & $\begin{array}{l}0.07- \\
1.21\end{array}$ & 0.089 & 0.38 & $\begin{array}{l}0.083- \\
1.70\end{array}$ & 0.204 \\
\hline $\begin{array}{l}\text { CR after } 2 \\
\text { cycles of } \\
\text { treatment }\end{array}$ & 0.53 & $0.23-1.24$ & 0.144 & & & & 0.71 & $\begin{array}{l}0.26- \\
1.92\end{array}$ & 0.495 & & & \\
\hline $\begin{array}{l}\text { OR after } 2 \\
\text { cycles of } \\
\text { treatment }\end{array}$ & 0.18 & $0.08-0.39$ & 0.000 & 0.67 & $\begin{array}{l}0.16- \\
2.83\end{array}$ & 0.584 & 0.25 & $\begin{array}{l}0.10- \\
0.62\end{array}$ & 0.003 & 0.20 & $\begin{array}{l}0.04- \\
1.16\end{array}$ & 0.073 \\
\hline $\begin{array}{l}\text { CR at the } \\
\text { end of } \\
\text { treatment }\end{array}$ & 0.32 & $0.15-0.66$ & 0.002 & 0.63 & $\begin{array}{l}0.24- \\
1.67\end{array}$ & 0.349 & 0.28 & $\begin{array}{l}0.11- \\
0.74\end{array}$ & 0.010 & 0.30 & $\begin{array}{l}0.08- \\
1.10\end{array}$ & 0.070 \\
\hline $\begin{array}{l}\text { OR at the } \\
\text { end of } \\
\text { treatment }\end{array}$ & 0.16 & $0.07-.033$ & 0.000 & 0.23 & $\begin{array}{l}0.05- \\
0.99\end{array}$ & 0.049 & 0.29 & $\begin{array}{l}0.12- \\
0.68\end{array}$ & 0.004 & 1.31 & $\begin{array}{l}0.024- \\
7.25\end{array}$ & 0.755 \\
\hline
\end{tabular}

\section{Discussion}

We retrospectively evaluated the efficacy, toxicity and cost of the R-M regimen, and compared it with the R-MA regimen. We found that the response rates and survival were not significantly different between the two regimens, indicating that the efficacy of the R-M regimen was comparable to the R-MA regimen. However, the R-M regimen yielded a higher chemotherapy completion rate with fewer severe toxicities, shorter LOS and lower cost than the R-MA regimen for patients with newly diagnosed PCNSLs.

Induction chemotherapy based on HD-MTX is considered as the standard approach for newly diagnosed PCNSLs. However, the best chemotherapeutic regimen remains unclear. Combinations with other chemotherapeutic agents have been shown to improve response and survival in patients with PCNSL, although few randomized clinical trials have supported this practice . $^{5}$ Controlled studies comparing the efficacy of different induction chemotherapy regimens were reviewed and are summarized 
in Appendix Table S1. However, comparisons were still challenging due to differing treatment schedules, consolidation strategies, and dosages and patient heterogeneity between the studies. It seems that adding more drugs into the HD-MTXbased chemotherapy regimens tends to improve the outcome but brings more toxicities. R-M regimen appears to be the combination chemotherapy with the least additional toxicities. Holdhoff et al. ${ }^{2}$ revealed that compared with the single HDMTX group, the R-M regimen improved CR rates and survival. In contrast, Kansara et al. ${ }^{3}$ reported that the addition of Rituximab to HD-MTX did not appear to improve outcomes. Both studies indicate that the addition of rituximab was feasible and well tolerated with overall minimal added toxicities. Our data suggest that the efficacy of the R-M regimen is comparable to that of the R-MA regimen, and most importantly, the R-M regimen shows the advantage of a better toxicity profile, although the sample size of the current study was small.

The combination of HD-Ara-C with HD-MTX has significantly improved outcomes, as described in large studies ${ }^{4,22}$. After the IELSG20 results were first published in $2009^{4}$, HD-Ara-C was widely applied in the management of PCNSLs. Recently, several studies reported the disappointing results of the HD-Ara-C containing regimens. One retrospective study demonstrated that neither PFS nor OS was higher in the Ara-C group than a therapeutic regimen without $A r a-C^{18}$. A randomized clinical trial (RCT) by Wu et al. ${ }^{15}$ showed that the non-Ara-C group (receiving the fotemustine, teniposide, and dexamethasone (FTD) regimen) had better apparent effects and safety than the Ara-C group (receiving the MA regimen). Hematological toxicities were the main concern. As expected, grade 3-4 hematological toxicities including neutropenia (90\%, 56\% and 59\%, respectively), thrombocytopenia ( $92 \%, 74 \%$ and $59 \%$, respectively) and anemia ( $46 \%, 36 \%$ and $23 \%$, respectively) were quite common in the IELSG20 trial, IELSG32 trial and the current study. Chemotherapy interruption due to toxicities occurred in $17.9 \%, 12 \%$ and $35.7 \%$ of patients in the IELSG20, IELSG32 and the current study, respectively. What's worse, a treatmentrelated mortality of 6-9\% was observed in these clinical trials. Thus, more effective and less toxic therapeutic regimens are urgently needed to balance therapy intensification with side effects, especially for old and weak patients. Our data suggest that the efficacy of the R-M regimen is comparable to that of the R-MA regimen with much fewer toxicities. We considered that the high frequency of grade 3-4 side effects with Ara-C might compromise the OS benefit. These toxicities were the main contributors to cost and prolonged hospital stay, resulting in a massive financial burden. In recent years, newly developed targeted drugs may provide new treatment options in PCNSL patients ${ }^{23}$. Ibrutinib (a selective covalent BTK inhibitor) ${ }^{24}$, nivolumab ${ }^{25}$ (a PD1 block) and lenalidomide (an immunomodulatory imide drug) ${ }^{26}$, showed promising response rates in refractory/recurrent PCNSL patients with good tolerability. we initiated a phase-2 trial, comparing the efficacy and safety of the R-M regimen with or without lenalidomide for newly-diagnosed PCNSLs, is ongoing (NCT04481815). As a result, we believe that in the rituximab era, HD-Ara-C may be eliminated from the initial combination chemotherapy regimen for PCNSLs, which warrants further evaluation in randomized trials.

There are two prognostic scoring systems developed specifically for PCNSLs. One is the International Extranodal Lymphoma Study Group (IELSG) scoring system, which divides patients into three different risk classes based on age, ECOG performance status, LDH, CSF total protein concentration, and involvement of deep brain structures ${ }^{27}$. The other is the Memorial SloanKettering Cancer Center (MSKCC) scoring system, which divides patients into three different risk classes, using age and $\mathrm{KPS}^{28}$. In our retrospective analysis, only $\mathrm{ECOG}>3$ was predictive of prognosis in multivariate analyses. Interestingly, we found that the early response and final response could be used for patient prognostication. Patients who achieved overall response within two cycles of therapy had an improved OS compared with those who did not, indicating a need for early treatment modification for those without early response in clinical practice. Patients who did not achieve CR at the end of therapy had a poor OS compared with those who did, indicating that treatment should be resumed for those without CR with consolidation (such as WBRT or autologous bone marrow transplantation) at the end of treatment.

Our study has several limitations given that it is a retrospective, single-institution analysis with modest sample size. First, the CSF protein data of $33 \%$ of patients were not available for the current study, similar to other retrospective studies. Therefore, it was impossible to determine different responses among IELSG subgroups. Second, it should be highlighted that only 3 of the 14 enrolled patients (21.4\%) in the R-MA group completed the entire protocol, which limited true judgments of its effectiveness. However, our study has multiple strengths. First, all our patients had a uniform, identical pathological 
diagnosis and received standardized treatment schedules, despite this being a retrospective study. Second, our singleinstitution retrospective study has one of the largest samples of patients with PCNSLs.

\section{Conclusion}

In conclusion, the efficacy of the R-M regimen is comparable to that of the R-MA regimen, but with fewer toxicities, well tolerability, shorter LOS and lower cost. Our results suggest that R-M regimen is an effective and well-tolerated combination treatment for PCNSLs, which warrants further evaluation in randomized trials.

\section{Declarations}

\section{Funding}

This work was supported by funds from Translational Research Grant of HCRCH (2020ZKZC01), the National Natural Science Foundation of China (No. 81830006), and Natural Science Foundation of Zhejiang Province of China (No. LY15H160038).

\section{Conflicts of Interest}

The authors declare that they have no conflict of interest.

\section{Availability of data and material}

All data generated or analyzed during this study are included in this published article.

\section{Code availability}

NA

\section{Author Contributions}

Xianggui Yuan and Teng Yu analyzed the patient data and wrote the manuscript; Huawei Jiang and Yurong Huang collected clinical data; Xiaohua Xu performed the statistical analysis; Yun Liang and Wenbin Qian designed and supervised the research project. All authors read and approved the final manuscript.

\section{Ethics approval}

This study was approved by the Human Ethics Committee of the Second Affiliated Hospital, School of Medicine, Zhejiang University, China.

\section{Consent to participate}

Informed consent was obtained from all participants included in the study.

\section{Consent to publish}

Patients signed informed consent regarding publishing their data and photograph.

\section{References}

1. Fox, C. P. et al. Guidelines for the diagnosis and management of primary central nervous system diffuse large B-cell lymphoma. Brit J Haematol 184, 348-363, doi:10.1111/bjh.15661 (2019).

2. Holdhoff, M. et al. High-dose methotrexate with or without rituximab in newly diagnosed primary CNS lymphoma. Neurology 83, 235-239, doi:10.1212/WNL. 0000000000000593 (2014). 
3. Kansara, R. et al. Rituximab with high-dose methotrexate in primary central nervous system lymphoma. American journal of hematology 90, 1149-1154, doi:10.1002/ajh.24204 (2015).

4. Ferreri, A. J. M. et al. High-dose cytarabine plus high-dose methotrexate versus high-dose methotrexate alone in patients with primary CNS lymphoma: a randomised phase 2 trial. Lancet (London, England) 374, 1512-1520, doi:10.1016/S0140-6736(09)61416-1 (2009).

5. Yang, H., Xun, Y., Yang, A. P., Liu, F. \& You, H. Advances and challenges in the treatment of primary central nervous system lymphoma. Journal of Cellular Physiology, doi:10.1002/jcp.29790 (2020).

6. Ferreri, A. J. et al. Chemoimmunotherapy with methotrexate, cytarabine, thiotepa, and rituximab (MATRix regimen) in patients with primary CNS lymphoma: results of the first randomisation of the International Extranodal Lymphoma Study Group-32 (IELSG32) phase 2 trial. Lancet Haemato/3, e217-227, doi:10.1016/S2352-3026(16)00036-3 (2016).

7. Coiffier, B. et al. CHOP chemotherapy plus rituximab compared with CHOP alone in elderly patients with diffuse large-Bcell lymphoma. The New England journal of medicine 346, 235-242 (2002).

8. Sun, X. et al. Methotrexate-cytarabine-dexamethasone combination chemotherapy with or without rituximab in patients with primary central nervous system lymphoma. Oncotarget 8, 49156-49164, doi:10.18632/oncotarget.17101 (2017).

9. Chen, C. et al. High-dose Methotrexate plus temozolomide with or without rituximab in patients with untreated primary central nervous system lymphoma: A retrospective study from China. Cancer Med 8, 1359-1367, doi:10.1002/cam4.1906 (2019).

10. Rubenstein, J. L. et al. Rituximab therapy for CNS lymphomas: targeting the leptomeningeal compartment. Blood 101, 466-468 (2003).

11. Batchelor, T. T. et al. Rituximab monotherapy for patients with recurrent primary CNS lymphoma. Neurology 76, 929-930, doi:10.1212/WNL.0b013e31820f2d94 (2011).

12. Bromberg, J. E. C. et al. Rituximab in patients with primary CNS lymphoma (HOVON 105/ALLG NHL 24): a randomised, open-label, phase 3 intergroup study. The Lancet. Oncology 20, 216-228, doi:10.1016/S1470-2045(18)30747-2 (2019).

13. Bromberg, J. E. C., van der Meulen, M. \& Doorduijn, J. K. The Role of Rituximab in Primary Central Nervous System Lymphoma. Current oncology reports 22, 78, doi:10.1007/s11912-020-00941-8 (2020).

14. Abrey, L. E. et al. Report of an international workshop to standardize baseline evaluation and response criteria for primary CNS lymphoma. Journal of clinical oncology: official journal of the American Society of Clinical Oncology 23, 5034-5043 (2005).

15. Wu, J. et al. Fotemustine, teniposide and dexamethasone versus high-dose methotrexate plus cytarabine in newly diagnosed primary CNS lymphoma: a randomised phase 2 trial. J Neuro-Oncol 140, 427-434, doi:10.1007/s11060-0182970-x (2018).

16. Ferreri, A. J. M. et al. Clinical relevance of the dose of cytarabine in the upfront treatment of primary CNS lymphomas with methotrexate-cytarabine combination. The oncologist 16, 336-341, doi:10.1634/theoncologist.2010-0361 (2011).

17. Fritsch, K. et al. High-dose methotrexate-based immuno-chemotherapy for elderly primary CNS lymphoma patients (PRIMAIN study). Leukemia 31, 846-852, doi:10.1038/leu.2016.334 (2017).

18. Wang, X.-x. et al. Clinical outcomes of patients with newly diagnosed primary central nervous system lymphoma are comparable on treatment with high-dose methotrexate plus temozolomide and with high-dose methotrexate plus cytarabine: a single-institution experience. Leukemia Lymphoma 55, 2497-2501, doi:10.3109/ 10428194.2014.889823 (2014).

19. Birnbaum, T., Stadler, E. A., von Baumgarten, L. \& Straube, A. Rituximab significantly improves complete response rate in patients with primary CNS lymphoma. J Neuro-Oncol 109, 285-291, doi:10.1007/s11060-012-0891-7 (2012).

20. Mocikova, H. et al. Role of rituximab in treatment of patients with primary central nervous system lymphoma: a retrospective analysis of the Czech lymphoma study group registry. Leukemia Lymphoma 57, 2777-2783 (2016).

21. Houillier, C. et al. Rituximab, methotrexate, procarbazine, vincristine and intensified cytarabine consolidation for primary central nervous system lymphoma (PCNSL) in the elderly: a LOC network study. J Neuro-Onco/ 133, 315-320,

Page $11 / 12$ 
doi:10.1007/ s11060-017-2435-7 (2017).

22. Ferreri, A. J. M. et al. A multicenter study of treatment of primary CNS lymphoma. Neurology 58, 1513-1520 (2002).

23. Mondello, P., Mian, M. \& Bertoni, F. Primary central nervous system lymphoma: Novel precision therapies. Critical reviews in oncology/hematology 141, 139-145, doi:10.1016/j.critrevonc.2019.06.009 (2019).

24. Grommes, C. et al. Ibrutinib Unmasks Critical Role of Bruton Tyrosine Kinase in Primary CNS Lymphoma. Cancer Discov 7, 1018-1029, doi:10.1158/2159-8290.CD-17-0613 (2017).

25. Nayak, L. et al. PD-1 blockade with nivolumab in relapsed/refractory primary central nervous system and testicular lymphoma. Blood 129, 3071-3073, doi:10.1182/blood-2017-01-764209 (2017).

26. Houillier, C. et al. Lenalidomide monotherapy as salvage treatment for recurrent primary CNS lymphoma. Neurology $\mathbf{8 4}$, 325-326, doi:10.1212/ WNL. 0000000000001158 (2015).

27. Ferreri, A. J. M. et al. Prognostic scoring system for primary CNS lymphomas: the International Extranodal Lymphoma Study Group experience. Journal of clinical oncology: official journal of the American Society of Clinical Oncology 21, 266-272 (2003).

28. Abrey, L. E. et al. Primary central nervous system lymphoma: the Memorial Sloan-Kettering Cancer Center prognostic model. Journal of clinical oncology: official journal of the American Society of Clinical Oncology 24, 5711-5715 (2006).

\section{Figures}

\section{Figure 1}

\section{Response, survival and cost by treatment group.}

(A)Response; (B)progression-free survival; (C)overall survival; (D)chemotherapy completion rate; (E)average length of hospital stay; (F)average total hospital cost per course per patient; (G)average antibiotic costs per course per patient.

\section{Figure 2}

\section{Kaplan-Meier survival curves.}

(A)Progression-free survival by subtypes (multifocal vs. monofocal); (B)progression-free survival by response ( $\geq$ PR vs. $<P R$ ) after 2 cycles of induction chemotherapy; (C)progression-free survival by response (CR vs. $<C R$ ) at the end of induction chemotherapy; (D)progression-free survival by response ( $\geq$ PR vs. $<P R$ ) at the end of induction chemotherapy; (E)overall survival by subtype (ECOG $>3$ vs.ECOG $\leq 3)$; (F)overall survival by response ( $\geq P R$ vs. $<P R$ ) after 2 cycles of induction chemotherapy; $(\mathrm{G})$ overall survival by response (CR vs. $<\mathrm{CR}$ ) at the end of induction chemotherapy; $(\mathrm{H})$ overall survival by response ( $\geq$ PR vs. $<P R$ ) at the end of induction chemotherapy.

\section{Supplementary Files}

This is a list of supplementary files associated with this preprint. Click to download.

- TableS1.SummaryoftheinductionchemotherapyregimensusedfornewlydiagnosedpatientswithPCNSLs.docx 